Pacific Journal of Mathematics

LIFTING PROJECTIONS OF CONVEX POLYHEDRA 


\section{LIFTING PROJECTIONS OF CONVEX POLYHEDRA}

\section{DaVid W. WALKuP AND Roger J.-B. Wets}

If $\tau$ is a projection of a closed convex polyhedron $P$ onto a convex polyhedron $Q$, then a lifting of $Q$ into $P$ is defined to be a single-valued inverse $\tau^{*}$ of $\tau$ such that $\tau^{*}(Q)$ is the union of closed faces of $P$. The main result of this paper, designated the Lifting Theorem, asserts that there always exists a lifting $\tau^{*}$, provided only that there exists at least one face of $P$ on which $\tau$ acts one-to-one. The lifting theorem represents a unifying generalization of a number of results in the theory of convex polyhedra and should prove useful as an investigative as well as a conceptual tool. In the course of the proof, a special case of the Lifting Theorem is translated into linear programming terms and stated as the Basis Decomposition Theorem, which summarizes the behavior of a linear program as a function of its right-hand sides. In particular, the fact that a lifting is necessarily a piecewise linear homeomorphism is reflected in the Basis Decomposition Theorem as the observation that the optimal solution of a linear program can always be chosen as a continuous function of the right-hand sides.

Definition. Suppose $\tau$ is an affine transformation taking a closed convex polyhedron $P$ onto a closed convex polyhedron $Q$. A function $\tau^{*}$ taking $Q$ into $P$ is said to be a lifting of $Q$ into $P$ relative to $\tau$ if

(i) $\tau^{*}$ is a single-valued inverse of $\tau$, that is, $\tau^{*}(q)$ is a point of $\tau^{-1}(q)$ for each $q \in Q$, and

(ii) $\tau^{*}(Q)$ is the union of closed faces of $P$.

A lifting is said to be regular (strictly regular) if there exists an affine functional $f$ on $P$ such that for each $q$ in $Q$ the image $\tau^{*}(q)$ is some point (the unique point) of $\tau^{-1}(q)$ at which $f$ is minimized.

Lifting THEOREM. Suppose $\tau$ is an affine transformation of the closed convex polyhedron $P$ onto the closed convex polyhedron $Q$ which acts one-to-one on some closed face $F$ of $P$. Then there exists a strictly regular lifting $\tau^{*}$ of $Q$ into $P$ relative to $\tau$ such that $F \subset \tau^{*}(Q)$.

The Lifting Theorem can be related to a number of topics in the theory of convex polyhedra which differ more or less in their basic viewpoint. First, the theorem is related to the theory of paths on the edges of polyhedra, as can be seen easily by considering the special case in which $Q$ is one-dimensional. Second, in [2, p. 168] Eggleston, Grünbaum, and Klee make use of the fact that if an affine transfor- 
mation $\tau$ takes a bounded convex polyhedron $P$ onto an $s$-dimensional polyhedron $Q$ then it takes the $s$-dimensional skeleton of $P$ onto $Q$. The Lifting Theorem is a generalization of this result, since every vertex of a bounded polyhedron $P$ is a face on which $\tau$ acts one-toone and since a lifting $\tau^{*}$ of $Q$ into $P$ necessarily takes $Q$ onto the union of $s$-dimensional faces of $P$. In fact, as will be seen from Proposition 3 , the Lifting Theorem replaces the sufficient condition that $P$ be bounded by the weaker necessary and sufficient condition that $\tau$ act one-to-one on the lineality space of $P$. Finally, we note that $\tau^{-1}(q)$ may be interpreted as one of a family of polyhedra whose defining halfspaces are subject to translations parametrized linearly by $q$. Viewed in this way the Lifting Theorem asserts in a rigorous way that the vertices of a polyhedron move continuously and piecewise linearly as its defining halfspaces are perturbed. The Lifting Theorem is used in this context in [4] to show that if the space of polyhedra $\tau^{-1}(q)$ is given the Hausdorff metric, then $\tau^{-1}$ is a Lipschitz function on $Q$. This result in turn can be used to establish some regularity conditions for convex programs with linear constraints [5].

The Basis Decomposition Theorem collects and sharpens certain results on a perturbation theory for linear programs which appeared in the Appendix of [6]. As already mentioned, the Basis Decomposition Theorem shows that it is always possible to make the optimal solution of a linear program a continuous function of its right-hand sides. This fact is used in [3]. The results of Dantzig, Folkman, and Shapiro [1], when applied to the very special case of a linear program, show that the set $M(t)$ of all optimal solutions of a program is an upper semicontinuous set-valued function of the right-hand sides $t$. They also show that, when restricted to that part of its domain on which it is single-valued, $M(t)$ is continuous. The basis Decomposition Theorem asserts the existence of a continuous single-valued function $m(t)$, sometimes known as a continuous selection, such that $m(t)$ is a member of $M(t)$ for all $t$. We remark that in general a continuous selection cannot be found if variation of other parameters of a linear program is considered.

Section 5 contains an example which may help the reader visualize some of the results obtained here, and which shows that certain of these results cannot be significantly sharpened.

2. Elementary properties of liftings. In addition to serving as an introduction to the concept of a lifting, the four elementary propositions of this section will be useful in the proofs of the theorems and in the applications of the Lifting Theorem mentioned in the Introduction. 
Proposition 1. If $\tau$ is an affine transformation of a polyhedron $P$ onto a polyhedron $Q$ and $\tau^{*}$ is a lifting of $Q$ into $P$ relative to $\tau$, then $\tau^{*}$ is a piecewise linear homeomorphism.

Proof. Of course $\tau^{*}$ is one-to-one and its inverse is continuous since it is a restriction of $\tau$. To show that $\tau^{*}$ is continuous we must show that $\tau(S)$ is closed in $Q$ for any relatively closed subset $S$ of $\tau^{*}(Q)$. Since $\tau^{*}(Q)$ is closed, so is $S$. The set $S$ is a finite union of sets $S_{i}=S \cap F_{i}$, where each $F_{i}$ is a closed face of $P$ in $\tau^{*}(Q)$. Since $F_{i}$ is in $\tau^{*}(Q), \tau$ acts one-to-one on $F_{i}$, and hence $\tau$ is a affine homeomorphism on $F_{i}$. If follows that $\tau\left(S_{i}\right)$ and therefore $\tau(S)=\bigcup_{i} \tau\left(S_{i}\right)$ is closed. This establishes that $\tau^{*}$ is a homeomorphism. The sets of the form $\tau(F)$, where $F$ is a maximal face of $P$ contained in $\tau^{*}(Q)$, cover $Q$ and are polyhedral regions of linearity of $\tau^{*}$.

Proposition 2. Suppose $\tau$ is an affine transformation of the finite-dimensional linear space $E$ onto a space $E^{\prime}$ taking a convex polyhedron $P$ onto a polyhedron $Q$. Suppose further that $F$ is any face of $P$ (including possibly $P$ itself), $p$ is a point in the relative interior of $F$, and $q=\tau(p)$. Then $\tau$ acts one-to-one on $F$ if and only if $p$ is a vertex of $\kappa(q)=\tau^{-1}(q) \cap P$.

Proof. By supposition $p$ is in the relative interior of $F$. Thus if $\tau$ does not act one-to-one on $F$ there is a line segment in $\tau^{-1}(q) \cap F$ passing through $p$. From this it is immediate that $p$ is not a vertex of $\kappa(q)=\tau^{-1}(q) \cap P$. Conversely, if $p$ is not a vertex of $\kappa(q)$, there is a line segment $l$ in $\kappa(q)$ passing through $p$. Since the face $F$ must contain every line segment in $P$ through $p, l$ must be contained in $\tau^{-1}(q) \cap F$, i.e., $\tau$ does not act one-to-one on $F$.

Proposition 3. Suppose $\tau$ is an affine transformation of the finite-dimensional linear space $E$ onto a space $E^{\prime}$ taking a convex polyhedron $P$ onto a polyhedron $Q$. Then the following are equivalent:

(i ) $\tau$ acts one-to-one on some face $F$ of $P$.

(ii) $\tau$ acts one-to-one on the lineality space of $P$.

(iii) For some (or alternatively every) $q$ in $Q$ the polyhedron $\kappa(q)=\tau^{-1}(q) \cap P$ has at least one vertex.

Proof. Items (i) and (ii) are equivalent since in any polyhedron there is a face which is a translate of the lineality space and every face contains a translate of the lineality space. The equivalence of (i) and (iii) is an easy consequence of the previous proposition.

Proposition 4. Suppose $\tau$ is an affine transformation of the 
finite-dimensional linear space $E$ onto a space $E^{\prime}$ taking a convex polyhedron $P$ onto a polyhedron $Q$ and $S$ is any polyhedron in $E^{\prime}$ intersecting $Q$. If $\tau^{*}$ is a lifting of $Q$ into $P$ relative to $\tau$, then the restriction of $\tau^{*}$ to $Q \cap S$ is a lifting of $Q \cap S$ into the polyhedron $\kappa(Q \cap S)=\tau^{-1}(Q \cap S) \cap P$ relative to the restriction of $\tau$ to $\kappa(Q \cap S)$. If $\tau^{*}$ is regular or strictly regular, so is its restriction to $Q \cap S$.

Proof. For each point $q$ in $E^{\prime}, \tau^{-1}(q)$ is a flat in $E$, and hence $S^{\prime}=\tau^{-1}(S)$ is a polyhedron in $E$ whose faces are of the form $\tau^{-1}(F)$, where $F$ is a face of $S$. Note that $\kappa(Q \cap S)$ is just $P \cap S^{\prime}$. It is trivial that the restriction of $\tau^{*}$ to $Q \cap S$ satisfies part (i) of the definition of a lifting. That is satisfies part (ii) of the definition follows from the fact that the faces of the intersection of two polyhedra, in particular $\kappa(Q \cap S)=S^{\prime} \cap P$, are exactly the nonempty intersections of the faces of each. If $\tau^{*}$ is regular or strictly regular with respect to the function $f$, then clearly the restriction of $\tau^{*}$ to $Q \cap S$ is regular or strictly regular with respect to the restriction of $f$ to $S^{\prime} \cap P$.

In $\S 5$ we shall prove a converse of Proposition 4 , specifically, that a strictly regular lifting $\tau^{*}$ of $Q \cap S$ into $\kappa(Q \cap S)$ can be extended to a lifting of $Q$ into $P$. However, it will be shown by counterexample that the condition of regularity on $\tau^{*}$ cannot be removed from the converse even if $S$ is a flat.

3. A theorem in linear programming. We begin by stating a lemma, which establishes a special case of the Strengthened Lifting Theorem to be proved in $\S 4$. Many of the details of this lemma are slightly strengthened forms of familiar results in the theory of linear programming; accordingly, parts of the proof are only a brief review of the necessary arguments. We follow the convention of writing pos $A$ for the set of column vectors spanned positively by the columns of a matrix $A$, i.e., the convex polyhedral cone $\{y \mid y=A x, x \geqq 0\}$.

LEMma. Let $P(c, t)$ denote the linear program

$$
\begin{array}{ll}
\min _{x} & c x \\
& A x=t \\
& x \geqq 0
\end{array}
$$

where $A$ is a fixed $m \times n$ matrix of rank $m$ whose columns are so ordered that the last $m$ of them constitute a feasible basis for $P\left(c^{0}, t^{0}\right)$ yielding an optimal solution $x^{0}$. Let $c(\theta)$ denote the cost vector whose ith component $c_{i}(\theta)$ is the sum of the ith component $c_{i}^{0}$ of $c^{0}$ and the 
ith power of $\theta$. If $P\left(c^{0}, t\right)$ has a unique solution $x^{*}(t)$ for all $t$ in $\operatorname{pos} A$, then $x^{*}$ is a lifting of pos $A$ into the positive orthant $R_{\tau}^{n}=$ $\{x \mid x \geqq 0\}$ relative to the transformation $\tau(x)=A x$ which is strictly regular with respect to the functional $f_{0}(x)=c^{0} x$. In any case there always exists $\theta^{*}>0$ such that:

(i) For all $\theta \in\left(0, \theta^{*}\right]$ the program $P(c(\theta), t)$ has a unique optimal solution $x^{*}(t)$ independent of $\theta$ for all $t$ in pos $A$, so that by the above, $x^{*}$ is a lifting of pos $A$ into $R_{+}^{n}$ which is strictly regular with respect to the functional $f_{\theta}(x)=c(\theta) x$.

(ii) $x^{*}(t)$ is a (not necessarily unique) optimal solution to $P\left(c^{0}, t\right)$ for all $t$ in pos $A$, i.e., $x^{*}$ is a regular lifting with respect to $f_{0}(x)=c^{0} x$.

(iii) $x^{*}\left(t^{0}\right)=x^{0}$.

Proof. Observe that, whatever the value of $c$, the program $P(c, t)$ is feasible if and only if $t$ lies in pos $A$. Since we assume $P\left(c^{0}, t^{0}\right)$ has an optimal feasible solution, the dual program is feasible. But feasibility of the dual is independent of $t$, hence $P\left(c^{0}, t\right)$ has an optimal basic feasible solution for all $t$ in pos $A$. Similarly, $P(c(\theta), t)$ must have an optimal basic feasible solution for all $t$ in pos $A$ and all positive $\theta$; for replacement of the quantities $c_{i}(\theta)$ by the lesser quantities $c_{i}^{0}=c_{i}(0)$ in a supposedly unbounded problem $P(c(\theta), t)$ would produce an unbounded problem $P\left(c^{0}, t\right)$.

There are finitely many bases in $A$, i.e., nonsingular $m \times m$ submatrices $A_{(k)}, 1 \leqq k \leqq K$. A basis $A_{(k)}$ satisfies the optimality criterion for $P(c(\theta), t)$ if the adjusted objective coefficients

$$
\bar{c}(\theta)=c(\theta)-c_{(k)}(\theta) A_{(k)}^{-1} A
$$

are nonnegative. A basis satisfies the strict optimality criterion if the nonbasic components of $\bar{c}(\theta)$ are strictly positive. (Here $c_{(k)}(\theta)$ denotes the basic part of $c(\theta)$, i.e., the subvector of $c(\theta)$ corresponding to the submatrix $A_{(k)}$ of $A$.) The components of $\bar{c}(\theta)$ are polynomials in $\theta$ and none of the nonbasic components are constant polynomials. Thus there exist $\theta_{k}>0$ such that either

(a) the basis $A_{(k)}$ fails to satisfy the optimality criterion for $P(c(\theta), t)$ for all $\theta \in\left(0, \theta_{k}\right]$, or

(b) the basis $A_{(k)}$ satisfies the strong optimality criterion for $P(c(\theta), t)$ for all $\theta \in\left(0, \theta_{k}\right)$.

If a basis is feasible and satisfies the strong optimality criterion, then it yields a unique optimal solution. (There can be other feasible bases also satisfying the strong optimality criterion, but only if the solution is degenerate.) Let $\theta^{*}$ be the minimum of the finite set of values $\theta_{k}$. It follows that for each $t$ in pos $A$ there is some feasible basis which 
satisfies the strong optimality criterion for $P(c(\theta), t)$ for all $\theta \in\left(0, \theta^{*}\right]$ and that this basis also satisfies the optimality criterion for $P\left(c^{0}, t\right)$. This establishes the first half of both parts (i) and (ii) of the lemma. Part (iii) of the lemma follows from the fact that when the basis consists of the last $m$ columns of $A$ the nonbasic component $\bar{c}_{j}(\theta)$ of $\bar{c}(\theta)$ is the sum of a nonnegative constant $\bar{c}_{j}=c_{j}^{0}-c_{(k)}^{0} A_{(k)}^{-1} A^{j}$ and a polynomial $\theta^{j}-\left(\theta^{n-m+1}, \cdots, \theta^{n}\right) A_{(k)}^{-1} A^{j}$ whose leading term (with smallest exponent) has a +1 coefficient, so that only case (b) above applies.

It remains to prove the first statement of the lemma, from which the rest of (i) and (ii) will follow. Thus suppose that the optimal solution $x^{*}(t)$ of $P\left(c^{0}, t\right)$ is unique for all $t$ in pos $A$. Clearly $x^{*}$ will be strictly regular with respect to $f_{0}$ if we show that it is a lifting, and just as clearly $x^{*}$ satisfies part $(i)$ of the definition of a lifting. To prove that $x^{*}$ satisfies part (ii) of the definition it suffices to show that if $F$ is the face of $R_{+}^{n}$ containing $x=x^{*}(t)$ in its relative interior and $x^{\prime}$ is any other point of $F$, then $x^{\prime}=x^{*}\left(t^{\prime}\right)$ for some $t^{\prime}$ in pos $A$. But $F$ is the set of vectors obtained from $x$ by independently multiplying its nonzero components by nonnegative scalars, and any such vector is clearly obtained by altering $t$ without affecting feasibility or optimality of the feasible, optimal basis yielding $x$.

The foregoing lemma has shown that the behavior of a linear program as a function of its right-hand sides can be summarized using the concept of a lifting. There is an additional concept that may be of value in visualizing this behavior. If $\tau^{*}$ is a lifting of a polyhedron $Q$ into a polyhedron $P$, then it is immediate that the image $\tau^{*}(Q)$ is the union of the cells of a finite closed polyhedral complex. By definition a finite closed polyhedral complex will be any finite collection $\mathscr{K}$ of closed convex polyhedra, called the cells of $\mathscr{K}$, such that: of $\mathscr{K}$.

(i) If $C$ is a cell of $\mathscr{K}$ then every closed face of $C$ is a member

(ii) If $C_{1}$ and $C_{2}$ are distinct cells of $\mathscr{K}$ then either they are disjoint, or one is a face of the other, or their intersection is a face of each.

Since the restriction of $\tau$ to the set $\tau^{*}(Q)$ is a homeomorphism and is linear on the faces of $P$ in $\tau^{*}(Q)$, it defines a decomposition of $Q$ into a finite closed polyhedral complex. For the special case described in the lemma the cells of the complex associated with $x^{*}(\operatorname{pos} A)$ are simplicical cones, that is, polyhedral cones affinely equivalent to orthants. Using these observations we may incorporate parts of the lemma in the following theorem, which constitutes an extension of certain of the results summarized in the Appendix of [6]. Expressing part (ii) of the lemma in this form it is, if possible, even clearer that the solution of a linear program can be made a continuous function 
of its right-hand sides.

Basis Decomposition Theorem. Let $P(t)$ denote the linear program

$$
\begin{array}{ll}
\min _{x} & c x \\
& A x=t \\
& x \geqq 0
\end{array}
$$

where $c$ is fixed and $A$ is a fixed $m \times n$ matrix of rank $m$. Then:

(i) $P(t)$ is feasible if and only if $t$ lies in the closed convex polyhedral cone pos $A$ of dimension $m$.

(ii) Either $P(t)$ is bounded for all $t$ in pos $A$ or $P(t)$ is unbounded for all $t$ in pos $A$.

(iii) If $P(t)$ is bounded there exist a decomposition of pos $A$ into a finite closed polyhedral complex $\mathscr{K}$ whose cells are simplicial cones with vertex at the origin and a one-to-one correspondence between the one-dimensional cells of $\mathscr{K}$ and selected columns of $A$ which generate them, such that

(a) the closed m-dimensional cells of $\mathscr{K}$ cover pos $A$ and

(b) the $m$ columns of $A$ associated with the edges of a closed $m$ dimensional cell $C$ of $\mathscr{K}$ constitute an optimal basis for all $t$ in $C$.

REMARK. At first glance one might guess that a decomposition of pos $A$ into simplicial cones could be obtained by selecting for each $t$ the optimal feasible basis which is lexicographically least with respect to some ordering on the columns of $A$. However, it can happen that a basis which is lexicographically preferred for some $t$ is not preferred for all $t^{\prime}$ for which it is feasible. Consequently the resulting "decomposition" need not be simplicial nor yield a continuous solution.

4. Proof of the Lifting Theorem. This section will be devoted exclusively to the proof of the following strengthened version of the Lifting Theorem stated in the Introduction.

Strengthened Lifting Theorem. Suppose $\tau$ is an affine transformation of a finite-dimensional linear space $E$ onto the linear space $E^{\prime}$ taking the closed convex polyhedron $P$ in $E$ onto a closed convex polyhedron $Q$ in $E^{\prime}$ and that $f_{0}$ is an affine functional on $E$ which achieves a (not necessarily unique) minimum on

$$
\kappa\left(q^{0}\right)=\tau^{-1}\left(q^{0}\right) \cap P
$$

at a vertex $p^{0}$ of $\kappa\left(q^{0}\right)$ for some $q^{0}$ in $Q$. If $f_{0}$ has a unique minimum on $\kappa(q)$ for all $q$ in $Q$, then $f_{0}$ defines a lifting $\tau^{*}$ of $Q$ into $P$ rela- 
tive to $\tau$ which is strictly regular with respect to $f_{0}$. In any case there exist a class of affine functions $f_{\theta}$ on $E$ parametrized continuously by $\theta$ on the interval $[0,1]$ and a lifting $\tau^{*}$ of $Q$ into $P$ relative to $\tau$ such that $\tau^{*}$ is strictly regular with respect to $f_{\theta}$ for $\theta \in(0,1]$, $\tau^{*}$ is regular with respect to $f_{0}$, and $\tau^{*}\left(q^{0}\right)=p^{0}$.

Proof. It is clear that we may as well suppose that $\tau$ is a linear projection of $E$ into itself taking the polyhedron $P$ onto the polyhedron $Q$ in the subspace $\tau(E)$. Let $N$ and $L$ denote respectively the nullspace of $\tau$ and the lineality space of $P$ (the largest subspace of $E$ for which a translate lies in $P$ ). The equivalence of parts (ii) and (iii) of Proposition 3 shows that $N \cap L=0$. Thus we may choose a subspace $E_{1}$ of $E$ such that $E_{1}, N$, and $L$ are independent and span $E$. The polyhedron $P$ is the sum of $L$ and a polyhedron $P^{\prime}=P \cap\left(E_{1}+\right.$ $N+q^{0}$ ) with a trivial lineality space. The projection $\tau$ takes $P^{\prime}$ onto a polyhedron $Q^{\prime}$ in $E_{1}+q^{0}$ such that $Q=Q^{\prime}+L$. (Thus $L$ is contained in, but need not be equal to, the lineality space of $Q$.) It is not difficult to see that to prove the theorem it is sufficient to prove it for $P^{\prime}, Q^{\prime}, p^{0}, q^{0}$, and the restrictions of $\tau$ and $f_{0}$ to $E_{1}+N+q^{0}$.

Now it is well known that any polyhedron $P^{\prime}$ with trivial lineality space is affinely equivalent to the intersection of the orthant $R_{+}^{n}$. and a $k$-dimensional flat $K$ in $R^{n}$, where $k$ is the dimension of $P^{\prime}, n$ is the number of $(k-1)$-dimensional faces of $P^{\prime}$, and $K$ intersects the relative interior of $R_{+}^{n}$. Clearly we may identify $P^{\prime}$ with $R_{+}^{n} \cap K$ and suppose that $\tau$ is an affine projection taking $K$, which is the affine hull of $P^{\prime}$, onto a subflat $S$ of $K$. It is then possible to extend $\tau$ to all of $R^{n}$ in such a way that the identity $\tau^{-1}(S)=K$ is preserved. The functional $f_{0}$ may be extended from $K$ to $R^{n}$ in any way; $p^{0}$ will still be a vertex of $\kappa\left(q^{0}\right)$ minimizing the (extended) functional $f_{0}$.

We next show that the lemma applies. Let $M$ be the flat $\tau\left(R^{n}\right)$ of dimension $m$, say, (so that $M \cap K=S$ ). With respect to a coordinate system in $M$ with origin at $\tau(0)$ the projection $\tau$ can be given by a matrix equation $\tau(x)=A x$, where $A$ is an $m \times n$ matrix of rank $m$. Let $F$ be the face of $R_{+}^{n}$ containing $p^{0}$ in its relative interior. Then $F$ is the set of vectors in $R_{+}^{n}$ whose components are zero in every position that $p^{0}$ has a zero component and nonnegative in every position that $p^{0}$ has a positive component. By Proposition 2, $\tau$ acts oneto-one on $F$. Hence there are at most $m$ nonzero components of $p^{0}$ and the corresponding columns of $A$ must be independent. Since $A$ has rank $m$ the coordinates of $R^{n}$ may be reordered if necessary so that the last $m$ columns of $A$ constitute a feasible basis for the equations $A x=t^{0}$, where $t^{0}=A p^{0}$, and of course this is an optimal basis with respect to the cost vector $c^{0}$, where $c^{0} x+d=f_{0}(x)$. Thus the 
lemma applies and we have a lifting $\tau^{*}$ and functionals

$$
f_{\theta}(x)=c(\theta) x+d \text {. }
$$

Since $\tau^{-1}(S)=M$ we may apply Proposition 4, and the restrictions of $\tau^{*}$ and $f_{\theta}$ to $Q^{\prime}=\tau\left(R_{+}^{n}\right) \cap S$ and $K$ respectively have all the properties required in the Strengthened Lifting Theorem.

4. A counterexample. The Lifting Theorem may be viewed as saying that a lifting of a subset of $Q$ onto a face $F$ of $P$ can be extended to a lifting of all $Q$. Thus it is perhaps natural to ask what more general partial liftings can always be extended. It is certainly clear that if a function $\tau^{*}$ defined on a subset $Q^{\prime}$ of $Q$ can be extended to a lifting of $Q$ into $P$ then it must be a single-valued inverse of $\tau$ taking $Q^{\prime}$ homeomorphically into the union of faces of $P$ on each of which $\tau$ acts one-to-one. Let us call a function $\tau^{*}$ satisfying these conditions a partial lifting. It is not difficult to find lowdimensional examples of partial liftings satisfying a number of additional plausible conditions for which no extension to $Q$ is possible. The following counterexample shows that a partial lifting $\tau^{*}$ need not have an extension even if $\tau^{*}$ takes a convex subset $Q^{\prime}$ of $Q$ onto the union of faces of $P$ on which $\tau$ acts one-to-one. It is necessary to go to dimension $d=4$ for $P$ to construct the counterexample, since an extension exists if the dimension of $Q$ is $0,1, d-1$, or $d$.

Consider the 3-dimensional trapezoidal prism $B$ in $R^{4}$ with vertices $a, b, \cdots, h$ listed in Figure 1 below. Figure 1 shows an accurate representation of the projection of $B$ into the plane of the first two coordinates. Let $P$ be the 4-dimensional pyramid with base $B$ and apex $p$. The 2 -faces of $p$ are the 2 -faces of $B$ plus the triangles with

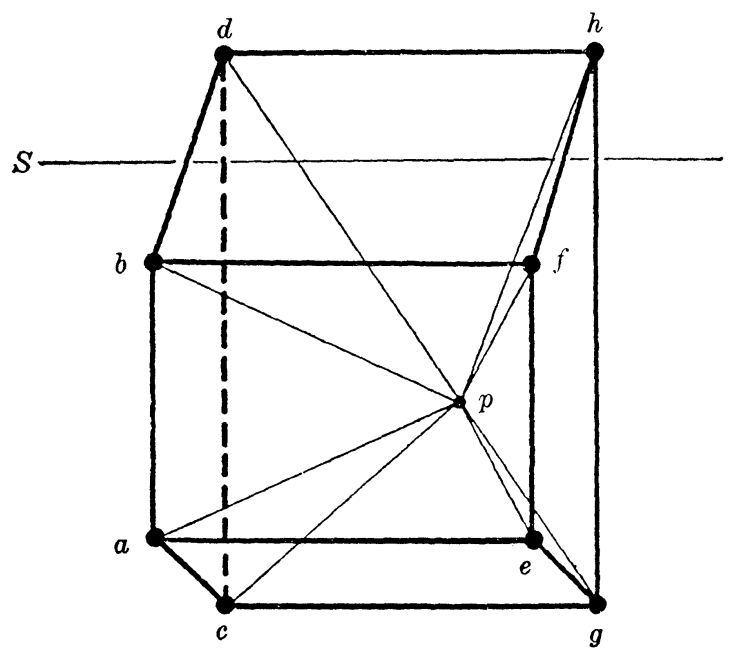

$$
\begin{aligned}
& a=(-4,-2, \quad 1,0) \\
& b=(-4, \quad 2, \quad 1,0) \\
& c=(-3,-3,-1,0) \\
& d=(-3, \quad 5,-1,0) \\
& e=(1,-2, \quad 1,0) \\
& f=(1,2, \quad 1,0) \\
& g=(2,-3,-1,0) \\
& h=(2, \quad 5,-1,0) \\
& p=(0, \quad 0, \quad 0,1)
\end{aligned}
$$


$P$ as apex and the 1-faces of $B$ as bases. Let $\tau$ be the coordinatewise projection sending $R^{4}$ onto the plane of the first two coordinates. It is readily seen that $\tau$ acts one-to-one on every face of $P$ of dimension 2 or less. Let $P^{\prime}$ be the union of the closed faces $c d p, d h p$, and $h f p$ of $P$, and let $Q^{\prime}$ be the image of $P^{\prime}$ under $\tau$. Then $Q^{\prime}$ is convex and there is a unique single-valued inverse $\tau^{*}$ of $\tau$ taking $Q^{\prime}$ onto $P^{\prime}$, but $\tau^{*}$ cannot be extended to a lifting of $Q$ into $P$. The difficulty is that any extension counterclockwise about $p$ from $c d p$ must involve $g c p$ and any extension clockwise about $p$ from $h f p$ must involve $f e p$.

The foregoing example may also be used as a counterexample to a converse of Proposition 4. Let $S$ be the line in the plane of Figure 1. There is a lifting of the line segment $Q \cap S$ into the 3-polyhedron $\tau^{-1}(Q \cap S) \cap P$ involving the intersection of $\tau^{-1}(S)$ and the faces $a b d c$, $c d p, d h p, h f p$, and fhge of $P$. But by the same arguments as above, this cannot be a restriction of a lifting of $Q$ into $P$.

We conclude with the following limited converse to Proposition 4.

Proposition 5. Suppose $\tau$ is an affine transformation taking a polyhedron $P$ onto a polyhedron $Q$. Suppose further that $S$ is a polyhedron intersecting $Q$, and $\tau_{1}^{*}$ is a lifting of $Q \cap S$ into $\tau^{-1}(Q \cap S)$ relative to $\tau$ which is strictly regular with respect to the affine functional $f_{1}$ defined on $\tau^{-1}(Q \cap S)$. Then there exists an extension $\tau^{*}$ of $\tau_{1}^{*}$ which is a lifting of $Q$ into $P$ relative to $\tau$ and which is strictly regular with respect to a functional $f$ on $P$. (But note that no relationship between $f_{1}$ and $f$ is asserted.)

Proof. Let $f_{0}$ be any extension of $f_{1}$ to $P$. If $q^{0}$ is any point of $Q \cap S$ then $f_{0}$ achieves its unique minimum on $\tau^{-1}\left(q^{0}\right)$ at the vertex $p^{0}=\tau_{1}^{*}\left(q^{0}\right)$. Now it may be impossible to choose $f_{0}$ so that it yields a strictly regular lifting of $Q$ into $P$, but the Strengthened Lifting Theorem assures us of a lifting $\tau^{*}$ of $Q$ into $P$ which is regular with respect to $f_{0}$ and strictly regular with respect to some functional $f$ close to $f_{0}$. We supposed that $\tau_{1}^{*}$ is strictly regular with respect to $f_{1}$, that is, $f_{1}$ has a unique minimum on $\tau^{-1}(q)$ for all $q$ in $Q \cap S$, hence $\tau^{*}$ must agree with $\tau^{*}$ on $Q \cap S$. Thus $\tau^{*}$ is a strictly regular extension of $\tau_{1}^{*}$.

\section{REFERENCES}

1. G. B. Dantzig, J. Folkman, and N. Shapiro, On the continuity of the minimum set of a continuous function, J. of Math. Anal. Appl. 17 (1967), 519-548.

2. H. G. Eggleston, B. Grünbaum, and V. Klee, Some semicontinuity theorems for convex polytopes and cell-complexes, Comment. Math. Helv. 39 (1964), 165-188. 
3. D. W. Walkup and R. J.-B. Wets, A note on decision rules for stochastic programs, Journal of Comp. and System Sciences, 2 (1968), to appear.

4. — A Lipschitzian characterization of convex polyhedra, Boeing document D1-82-0728, Boeing Scientific Research Laboratories, 1968.

5. - Some practical regularity conditions for nonlinear programs, Boeing document D1-82-0792, Boeing Scientific Research Laboratories, 1968.

6. R. J.-B. Wets, Programming under uncertainty: the equivalent convex program, SIAM J. Appl. Math. 14 (1966), 89-105.

Received June 12, 1968.

Boeing Scientific Research Labs 



\section{PACIFIC JOURNAL OF MATHEMATICS}

\section{EDITORS}

H. ROYDEN

Stanford University

Stanford, California

\section{R. $R$ PHELPS}

University of Washington

Seattle, Washington 98105

\section{J. DugunduI}

Department of Mathematics

University of Southern California

Los Angeles, California 90007

RICHARD ARENS

University of California

Los Angeles, California 90024

\section{ASSOCIATE EDITORS}

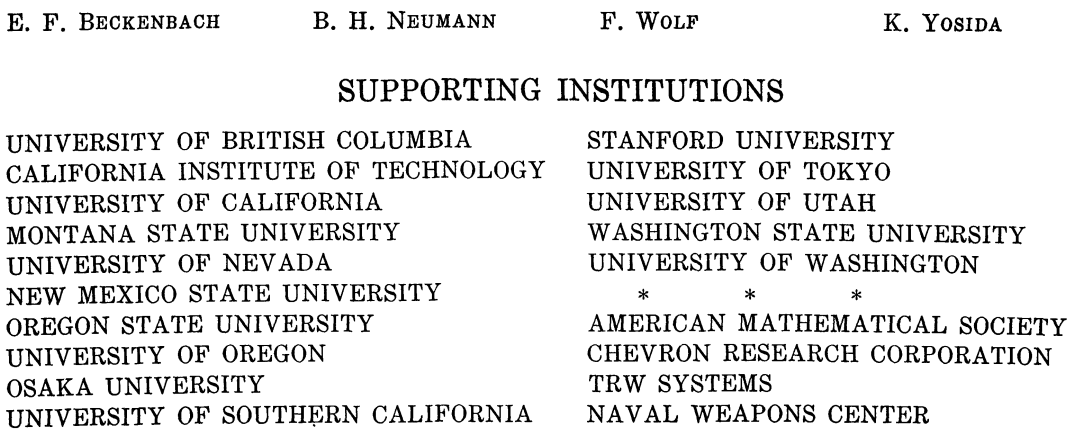

The Supporting Institutions listed above contribute to the cost of publication of this Journal, but they are not owners or publishers and have no responsibility for its content or policies.

Mathematical papers intended for publication in the Pacific Journal of Mathematics should be in typed form or offset-reproduced, double spaced with large margins. Underline Greek letters in red, German in green, and script in blue. The first paragraph or two must be capable of being used separately as a synopsis of the entire paper. It should not contain references to the bibliography. Manuscripts, in duplicate if possible, may be sent to any one of the four editors. Please classify according to the scheme of Math. Rev. 36, 1539-1546. All other communications to the editors should be addressed to the managing editor, Richard Arens, University of California, Los Angeles, California, 90024.

50 reprints are provided free for each article; additional copies may be obtained at cost in multiples of 50 .

The Pacific Journal of Mathematics is published monthly. Effective with Volume 16 the price per volume (3 numbers) is $\$ 8.00$; single issues, $\$ 3.00$. Special price for current issues to individual faculty members of supporting institutions and to individual members of the American Mathematical Society: $\$ 4.00$ per volume; single issues $\$ 1.50$. Back numbers are available.

Subscriptions, orders for back numbers, and changes of address should be sent to Pacific Journal of Mathematics, 103 Highland Boulevard, Berkeley, California, 94708.

PUBLISHED BY PACIFIC JOURNAL OF MATHEMATICS, A NON-PROFIT CORPORATION

Printed at Kokusai Bunken Insatsusha (International Academic Printing Co., Ltd.), 7-17, Fujimi 2-chome, Chiyoda-ku, Tokyo, Japan. 


\section{Pacific Journal of Mathematics}

Vol. 28, No. $2 \quad$ April, 1969

Richard Arens and Donald George Babbitt, The geometry of relativistic

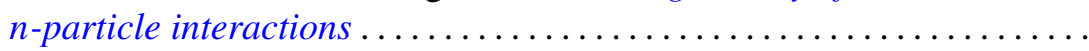

Kirby Alan Baker, Hypotopological spaces and their embeddings in lattices

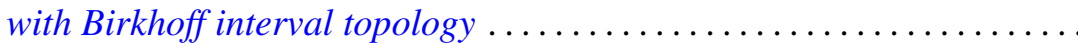

J. Lennart (John) Berggren, Finite groups in which every element is conjugate to its inverse ........................... 289

Beverly L. Brechner, Homeomorphism groups of dendrons . . . . . . . . . . . 295

Robert Ray Colby and Edgar Andrews Rutter, QF - 3 rings with zero singular ideal ................................. 303

Stephen Daniel Comer, Classes without the amalgamation property....... 309

Stephen D. Fisher, Bounded approximation by rational functions ......... 319

Robert Gaines, Continuous dependence for two-point boundary value problems..................................... 327

Bernard Russel Gelbaum, Banach algebra bundles ............... 337

Moses Glasner and Richard Emanuel Katz, Function-theoretic degeneracy criteria for Riemannian manifolds ...................... 351

Fletcher Gross, Fixed-point-free operator groups of order $8 \ldots \ldots \ldots \ldots 357$

Sav Roman Harasymiv, On approximation by dilations of distributions . . . . 363

Cheong Seng Hoo, Nilpotency class of a map and Stasheff's criterion ... . . 375

Richard Emanuel Katz, A note on extremal length and modutus.......... 381

H. L. Krall and I. M. Sheffer, Difference equations for some orthogonal polynomials ................................

Yu-Lee Lee, On the construction of lower radical properties ........... 393

Robert Phillips, Liouville's theorem........................... 397

Yum-Tong Siu, Analytic sheaf cohomology groups of dimension $n$ of

n-dimensional noncompact complex manifolds ..... . .

Michael Samuel Skaff, Vector valued Orlicz spaces. II...

James DeWitt Stein, Homomorphisms of $B^{*}$-algebras .... . .

Mark Lawrence Teply, Torsionfree injective modules .... . . .

Richard R. Tucker, The $\delta^{2}$-process and related topics. II .

David William Walkup and Roger Jean-Baptiste Robert Wets, Lifting

projections of convex polyhedra...

Thomas Paul Whaley, Large sublattices of a lattice. 